\title{
Mutation spectrum of TP53 gene predicts clinicopathological features and survival of gastric cancer
}

\author{
Tomomitsu Tahara ${ }^{1}$, Tomoyuki Shibata1, Yasuyuki Okamoto², Jumpei Yamazaki ${ }^{3}$, \\ Tomohiko Kawamura ${ }^{1}$, Noriyuki Horiguchi' ${ }^{1}$, Masaaki Okubo', Naoko Nakano ${ }^{1}$, \\ Takamitsu Ishizuka ${ }^{1}$, Mitsuo Nagasaka ${ }^{1}$, Yoshihito Nakagawa ${ }^{1}$, Naoki Ohmiya ${ }^{1}$ \\ ${ }^{1}$ Department of Gastroenterology, Fujita Health University School of Medicine, Toyoake, Japan \\ ${ }^{2}$ Department of Gastroenterology and Metabolism, Nagoya City University Graduate School of Medical Sciences, Nagoya, Japan \\ ${ }^{3}$ Laboratory of Molecular Medicine, Hokkaido University Graduate School of Veterinary Medicine, Sapporo, Japan \\ Correspondence to: Tomomitsu Tahara, email: tomomiccyu@yahoo.co.jp \\ Keywords: TP53 mutation, gastric cancer, spectrum, survival, hotspot mutations \\ Received: October 24, $2015 \quad$ Accepted: April 18, $2016 \quad$ Published: June 01, 2016
}

\section{ABSTRACT}

Background and aim: TP53 gene is frequently mutated in gastric cancer (GC), but the relationship with clinicopathological features and prognosis is conflicting. Here, we screened TP53 mutation spectrum of 214 GC patients in relation to their clinicopathological features and prognosis.

Results: TP53 nonsilent mutations were detected in 80 cases $(37.4 \%)$, being frequently occurred as C:G to T:A single nucleotide transitions at 5'-CpG-3' sites. TP53 mutations occurred more frequently in differentiated histologic type than in undifferentiated type in the early stage $(48.6 \%$ vs. $7 \%, P=0.0006)$, while the mutations correlated with venous invasion among advanced stage $(47.7 \%$ vs. $20.7 \%$, $P=0.04)$. Subset of GC with TP53 hot spot mutations (R175, G245, R248, R273, R282) presented significantly worse overall survival and recurrence free survival compared to others (both $P=0.001$ ).

Methods: Matched biopsies from GC and adjacent tissues from 214 patients were used for the experiment. All coding regions of TP53 gene (exon2 to exon11) were examined using Sanger sequencing.

Conclusion: Our data suggest that GC with TP53 mutations seems to develop as differentiated histologic type and show aggressive biological behavior such as venous invasion. Moreover, our data emphasizes the importance of discriminating TP53 hot spot mutations (R175, G245, R248, R273, R282) to predict worse overall survival and recurrence free survival of GC patients.

\section{INTRODUCTION}

Gastric cancer (GC) is one of the most common malignancies worldwide, accounted for approximately 70,000 new cases and 650,000 deaths per year $[1,2]$. Despite advances in diagnosis technologies, many patients still have advanced disease at diagnosis and treatment outcomes for such patients are poor [3]. Moreover, GC is heterogeneous in its clinical course and prognosis. However, underlying mechanisms to address this issue has not been fully understood.

Concerning the molecular abnormality in GC, several genetic changes in gastric carcinogenesis have been elucidated including oncogenes (KRAS, b-catenin, ERBB2, PIK3CA etc.) [4-7] and tumor-suppressor genes (CDH1, p16, TP53, ARID1A etc.) [8-11]. TP53 is the most studied gene in many cancers and may be possible candidate biomarker for GC. The gene encoding TP53 is located on chromosome $17 \mathrm{p}$ and consists of 11 exons and 10 introns. TP53 protein has important cellular functions, including in cell cycle regulation, apoptosis, and DNA repair $[12,13]$. Even by advances in new technologies, such as whole exome sequencing, TP53 is confirmed as the most frequently mutated gene in human cancers, with alterations occurring in about half of human cancers [8-11]. 
Concerning the association between TP53 status and subtypes and prognosis of GC, conflicting results did not conclude the clinical significance of TP53 as a molecular marker [14-17]. We reasoned that conflicting result may be partly due to the limitation of immunohistochemistry assay that may not detect truncating type mutations (frameshift and nonsense mutations) [14], or a lack of discriminations of different types of mutation occurring throughout the coding exons. To resolve these issues, we investigated TP53 mutation spectrum throughout the entire exons in $214 \mathrm{GC}$ cases. We aimed to clarify the association between clinicopathological features and prognosis of GC and TP53 mutation status with an attempt to their change types.

\section{RESULTS}

\section{Spectrum of TP53 mutations}

We performed Sanger sequencing of TP53 genes in 214 GC tissues and matched normal tissues Representative sequencing chromatograms are shown in Figure 1.

We found 80 cases $(37.4 \%)$ with TP53 nonsilent mutations. One tumor had 3 mutations, 3 had 2 mutations, and 76 had a single mutation, for a total of 85 mutations in this cohort. Mostly, these mutations were predicted to either truncate the protein through base substitutions, resulting in a stop codon (nonsense, 13 mutations) or a frame shift (16 mutations), multiple nucleotide polymorphism (1 mutation) or to damage the protein as predicted by SIFT analysis (52 out of 55 missense mutations) (Figure 2, Supplementary Table 2). These mutations were distributed across exon4 to exon10, but majority were in the DNA binding domain of central core region (Figure 3). Concerning the spectrum and nucleotide contexts of the substitution mutations, most frequent alterations were $\mathrm{C}: \mathrm{G}$ to $\mathrm{T}$ :A transitions (54.4\%), predominantly occurred at 5'-CpG-3'dinucleotide sites (47.1\%, Table 1).

\section{Association between TP53 mutations and clinicopathological subtypes of GC}

We investigated whether TP53 mutation positivity is associated with any clinicopathological subtypes of GC. Age, gender, location, H. pylori status, staging, histology, venous and lymphatic invasion, lymph node, liver, peritoneal and other distant metastasis were included for this analysis. Although we did not find any association between TP53 mutation positivity and age, gender, location, H. pylori status and staging (Table 2), TP53 mutation was more frequent in differentiated histologic type, compared to undifferentiated type (44.0\% vs. $26.9 \%$, $P=0.01)$. Notably, this association was more striking among early stage GC (T1, 48.6\% vs. $7 \%, P=0.0006)$ but not in advanced stage (T2 $<, 35.0 \%$ vs. $36.4 \%, P>0.1$ ) (Table 3). We also found that in advanced stage GC $(\mathrm{T} 2<)$, TP53 mutation was more frequent in cases having

\section{GC12}

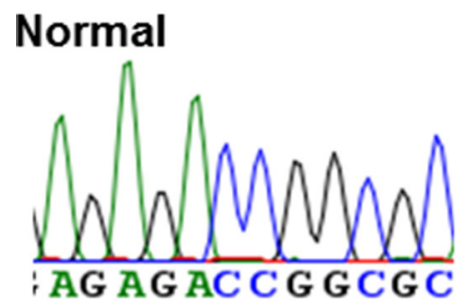

Normal

AG AG ACCG GCGC

\section{GC109}

Normal

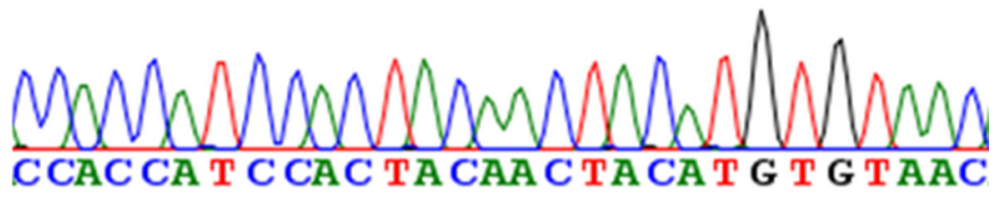



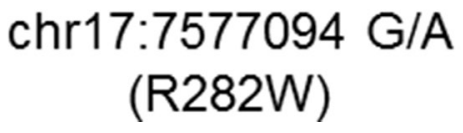

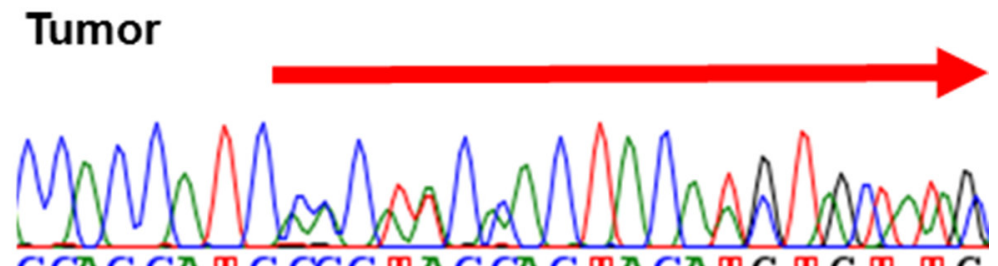

CCAC CA T C CCC TACCACTACA T G T G T G
Figure 1: Representative sequencing chromatograms of two cases with TP53 mutations. Below indicates the position at chromosome. Position at codon was indicated in the parentheses (left). 
venous invasion (47.7\% vs. $20.7 \%, P=0.04)$. A similar trend was also found in cases having more severe lymph node metastasis (N0 1 vs. N2 , 31.3\% vs. 47.1\%, $P<0.1$ ) (Table 4).

\section{Association between TP53 hot spot mutations and clinicopathological subtypes of GC}

Next we aimed to investigate whether TP53 mutation is associated with survival and relapse of GC patients. 210 cases were evaluated for this analysis. We did not find any significant association between overall survival and recurrence free survival among cases with or without TP53 mutation (data not shown). However, focusing on TP53 mutation hot spots within the central core (R175, G245, R248, R273, R282), which is reported to be mutated across various cancers. We found that the cases having mutation in this hot spots $(6.2 \%$, $\mathrm{n}=13$ ) presented significantly worse overall survival and recurrence free survival compared to others (both

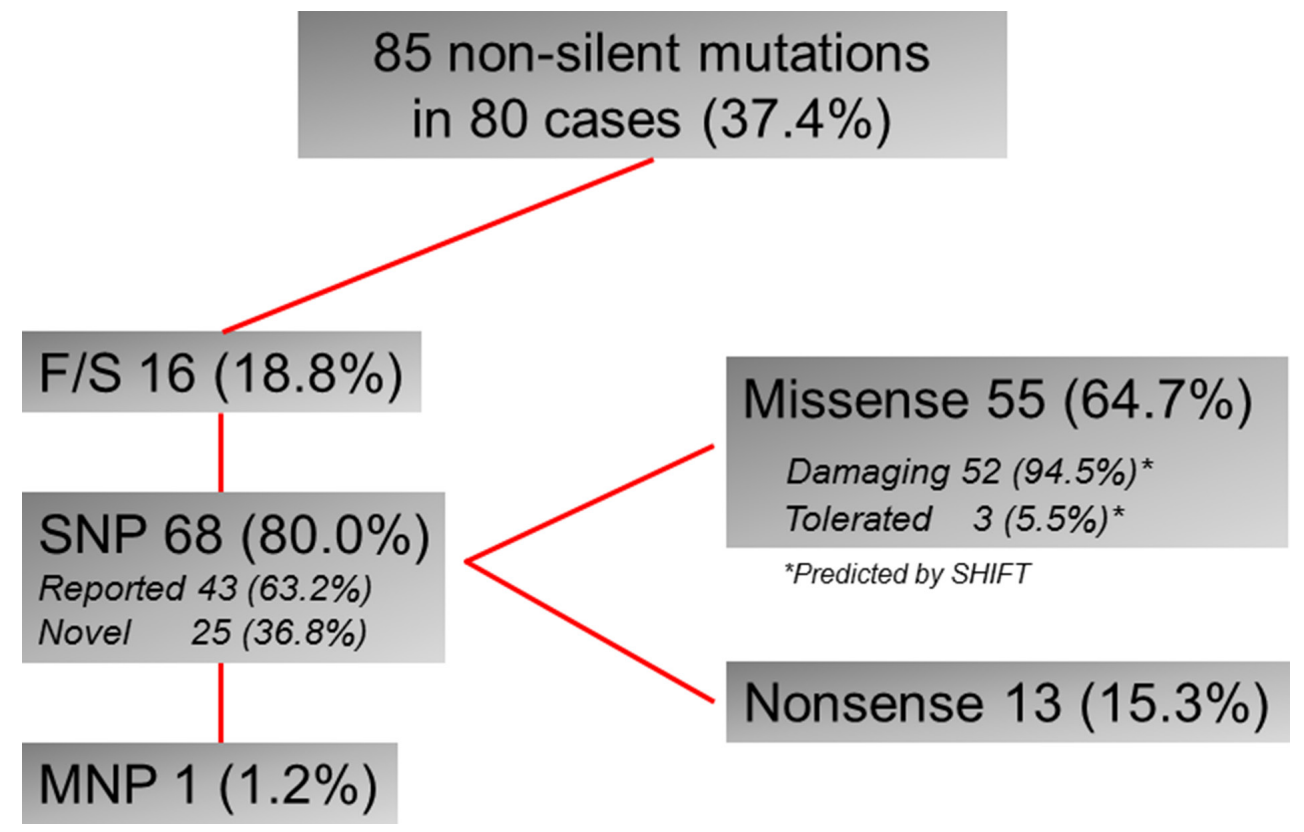

Figure 2: Summary of TP53 mutations in 214 GC cases. F/S, frame shift; SNP, single nucleotide polymorphism; MNP multiple nucleotide polymorphism;

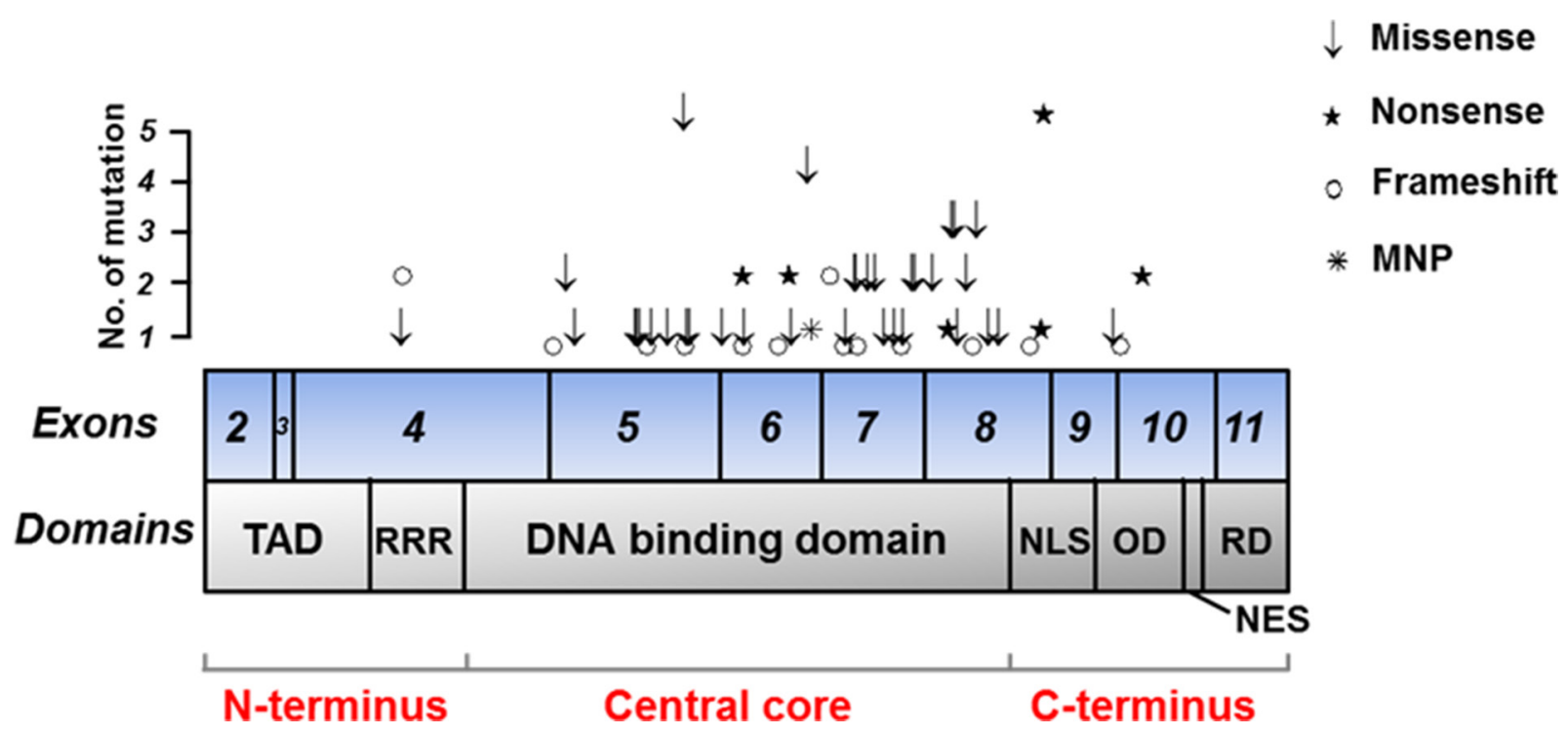

Figure 3: Mutation spectrum of TP53 in 214 GC cases. Individual exons are represented as numbered boxes. TAD, Trans activation domain; RRR, Proline-rich region; NLS, nuclear localization signal sequence; OD, Tetramerization domain; NES, nuclear export signal sequence; RD, Regulatory domain; 
Table 1: Spectrum of TP53 single base substitutions

\begin{tabular}{lc}
\hline Variables & n (\%) \\
\hline Total number of substitutions & 68 \\
Substitutions at C:G base pairs & $37(54.4)$ \\
$\mathrm{C}: \mathrm{G} \rightarrow \mathrm{T}: \mathrm{A}$ & $3(4.4)$ \\
$\mathrm{C}: \mathrm{G} \rightarrow \mathrm{G}: \mathrm{C}$ & $8(11.7)$ \\
$\mathrm{C}: \mathrm{G} \rightarrow \mathrm{A}: \mathrm{T}$ & \\
Substitutions at T:A base pairs & $9(13.2)$ \\
$\mathrm{T}: \mathrm{A} \rightarrow \mathrm{C}: \mathrm{G}$ & $3(4.4)$ \\
$\mathrm{T}: \mathrm{A} \rightarrow \mathrm{G}: \mathrm{C}$ & $6(8.8)$ \\
$\mathrm{T}: \mathrm{A} \rightarrow \mathrm{A}: \mathrm{T}$ & \\
Substitutions at specific dinucleotides $\$$ & $32(47.1)$ \\
5'-CpG-3' & $12(17.6)$ \\
5'-TpC-3' & \\
\hline
\end{tabular}

Base substitutions in coding sequences resulting in nonsynonymous changes are included (see Figure 2).

$\$$ Includes substitutions at the $\mathrm{C}$ or $\mathrm{G}$ of the 5'-CpG-3' dinucleotide, the $\mathrm{C}$ of the 5'-TpC-3' dinucleotide, or the $\mathrm{G}$ of the 5'GpA-3' dinucleotide.

Table 2: TP53 mutation status and subtypes of GC

\begin{tabular}{|c|c|c|}
\hline Variables n (\%) & TP53 wild type & TP53 mutated \\
\hline Age (mean +/- SEM) & $64.4+/-1.0$ & $66.4+/-1.4$ \\
\hline \multicolumn{3}{|l|}{ Gender } \\
\hline Female n $(\%)$ & $46(67.6)$ & $22(32.4)$ \\
\hline Male n $(\%)$ & $88(60.3)$ & $58(39.7)$ \\
\hline \multicolumn{3}{|l|}{ Location } \\
\hline Cardia n (\%) & $7(70.0)$ & $3(30.0)$ \\
\hline Upper n $(\%)$ & $19(65.5)$ & $10(34.5)$ \\
\hline Middle n (\%) & $70(64.8)$ & $38(35.2)$ \\
\hline Lower n (\%) & $38(56.7)$ & $29(43.3)$ \\
\hline \multicolumn{3}{|l|}{ H. pylori status } \\
\hline Negative n $(\%)$ & $19(65.5)$ & $10(34.5)$ \\
\hline Positive $\mathrm{n}(\%)$ & $115(62.2)$ & $70(37.8)$ \\
\hline \multicolumn{3}{|l|}{ Stage } \\
\hline $\operatorname{In}(\%)$ & $70(66.0)$ & $36(34.0)$ \\
\hline II n (\%) & $20(64.5)$ & $11(35.5)$ \\
\hline III n $(\%)$ & $21(58.3)$ & $15(41.7)$ \\
\hline IV n $(\%)$ & $22(55.0)$ & $18(45.0)$ \\
\hline \multicolumn{3}{|l|}{ Depth } \\
\hline $\mathrm{T} 1 \mathrm{n}(\%)$ & $62(63.3)$ & $36(36.7)$ \\
\hline $\mathrm{T} 2 \mathrm{n}(\%)$ & $16(66.7)$ & $8(33.3)$ \\
\hline T3 n (\%) & $8(80.0)$ & $2(20.0)$ \\
\hline $\mathrm{T} 4 \mathrm{n}(\%)$ & $48(58.5)$ & $34(41.5)$ \\
\hline
\end{tabular}

Stage was determined for 213 cases 
Table 3: TP53 mutation status and histologic subtypes of GC

\begin{tabular}{lcc}
\hline Variables n (\%) & TP53 wild type & TP53 mutated \\
\hline Total number of patients & $134(62.6)$ & $80(37.4)$ \\
All subjects $(n=214) \#$ & & \\
Differentiated n (\%) & $61(56.0)$ & $48(44.0)$ \\
Un differentiated n (\%) & $68(73.1)$ & $25(26.9)$ \\
Mixed n (\%) & $5(41.7)$ & $7(58.3)$ \\
Early stage (T1, $n=98) \$$ & & \\
Differentiated n (\%) & $35(51.5)$ & $33(48.5)$ \\
Un differentiated n (\%) & $26(92.9)$ & $2(7.1)$ \\
Mixed n (\%) & $1(50.0)$ & $1(50.0)$ \\
Advanced stage (T2 4, $n=116)$ & & $14(35.0)$ \\
Differentiated n (\%) & $26(65.0)$ & $24(36.4)$ \\
Un differentiated n (\%) & $42(63.6)$ & $6(60.0)$ \\
Mixed n $(\%)$ & $4(40.0)$ & \\
\hline
\end{tabular}

\#, $P=0.01 ; \$, P=0.0006$;

Table 4: TP53 mutation status and lymphatic and venal invasion, and metastasis in advanced GC

\begin{tabular}{lcc}
\hline & TP53 wild type & TP53 mutated \\
\hline Lymphatic invasion & $4(80.0)$ & $1(20.0)$ \\
Negative & $42(61.8)$ & $26(38.2)$ \\
Positive & & \\
Venous invasion\# & $23(79.3)$ & $6(20.7)$ \\
Negative & $23(52.3)$ & $21(47.7)$ \\
Positive & & \\
Lymphnode metastasis\$ & $44(68.8)$ & $20(31.3)$ \\
N0 1 & $27(52.9)$ & $24(47.1)$ \\
N2 & & $29(34.1)$ \\
Peritoneal dissemination & $56(65.9)$ & $15(50.0)$ \\
Negative & $15(50.0)$ & \\
Positive & & $42(38.9)$ \\
Liver metastasis & $66(61.1)$ & $2(28.6)$ \\
Negative & $5(71.4)$ & \\
Positive & & $41(38.3)$ \\
Other distant metastasis & $66(61.7)$ & $3(37.5)$ \\
Negative & $5(62.5)$ & \\
Positive & & \\
\hline
\end{tabular}

Lymphatic and venous invasion was determined for 73 cases

Metastasis was determined for 115 cases

$\#, P=0.04 ; \$, P<0.10$; 
$\mathrm{P}$ values $=0.001$, Figure 4). To confirm this result, we next conducted multivariate survival analysis using Cox's regression model to see whether the TP53 hotspot mutations would be associated with worse overall survival as an independent factor. Age, gender, histology, depth, lymph node metastasis, peritoneal dissemination, liver or other distant metastasis, staging and TP53 hotspot mutations were included for this analysis. The result demonstrated that TP53 hotspot mutations was associated with worse overall survival as an independent factor (hazard ratio $=3.57,95 \%$ confidence interval $=1.44-8.86$, $\mathrm{P}=0.006$, Table 5).

We also tried to investigate the association between TP53 hotspot mutations and recurrences of GC including liver, peritoneal, lymph node and others. In advanced GC $(\mathrm{T} 2<)$, this analysis showed that the cases that have TP53 hotspot mutations presented significantly higher liver or other recurrences compared to others $(P<0.05,0.02$, respectively) (Table 6).

We also evaluated whether the change types (nonsense, missense or frame shift), nucleotide contexts of the substitution mutations, and locations (exons, 5'-CpG3' sites 5'-TpC-3') of TP53 mutations would be associated with prognosis of GC. But association were all negative except for the mutations in the hot spots (data not shown).

\section{DISCUSSION}

Regarding the spectrum of TP53 mutations in our study, C:G to T:A transitions were predominantly occurred at 5'-CpG-3'dinucleotide sites, which was in line with other study in GC $[8,10,14]$. Not only the TP53 gene, $\mathrm{C}: \mathrm{G}$ to $\mathrm{T}: \mathrm{A}$ transitions are known to be most frequent transitions throughout the genomes in $\mathrm{GC}[8,10]$. C to $\mathrm{T}$ transition is induced by nitric oxide $[19,20]$, a substance known to be induced by H. pylori infections. C:G to T:A transitions are also specifically induced by N-methyl$\mathrm{N}$ '-nitro-N-nitrosoquanidine and $\mathrm{N}$-nitroso compounds found in foods, substances considered to be carcinogens in GC [21]. These foods are commonly consumed in high risk populations for developing GC including Japanese. Our result confirmed that $\mathrm{C}: \mathrm{G}$ to $\mathrm{T}: \mathrm{A}$ transitions are typical mutation spectrum of TP53 in GC, reflecting interaction between $H$. pylori infection and environmental predisposing factors.

There are conflicting results with respect to the prevalence of TP53 mutations as well as their relationship to clinicopathological features in GC [14], which may be partly due to difference of patient constitutions or the method for detection of mutations. Immunohistochemistry assay used in many studies lacks the ability to detect truncating type mutations (frameshift and nonsense mutations). Single strand conformation polymorphism (SSCP) analysis also misses considerable percentage of mutations [14]. In this study, all coding exons of TP53 gene were screened by the Sanger sequencing. TP53 mutation frequency in our dataset was $37.4 \%$, which was quite similar to that of recent Japanese study using the Digital PCR targeted sequencing coupled with macrodissection [11]. Other strength of our study was to use endoscopic biopsy specimens. Since the mutation detection rate seemed to be reliable even by using tiny

\section{-.L Hot spot mutations \\ + Wild type \\ - Other mutations}


Figure 4: Association between TP53 mutation and overall survival (left) and recurrence free survival (right) in GC patients. Statistical analysis was performed by the Log rank test. 
Table 5: Multivariate survival analysis using Cox's regression model for adjustment of clinicopathological factors and TP53 hotspot mutations

\begin{tabular}{lcc}
\hline Variables & HR (95\%CI) & $\boldsymbol{P}$ \\
\hline Age & $1.02(1.00-1.05)$ & 0.05 \\
Gender (male) & $1.74(0.88-3.16)$ & 0.11 \\
H. pylori positive & $0.81(0.32-2.08)$ & 0.66 \\
Location (cardia) & $3.61(1.22-10.65)$ & 0.02 \\
Histology (un differentiated type) & $1.11(0.57-2.17)$ & 0.11 \\
Depth & $2.12(1.08-4.13)$ & 0.03 \\
Lymph node metastasis & $1.69(0.72-3.95)$ & 0.23 \\
Peritoneal dissemination & $2.86(0.81-10.1)$ & 0.1 \\
Liver metastasis & $1.92(0.36-10.37)$ & 0.45 \\
Other distant metastasis & $1.45(0.45-4.65)$ & 0.53 \\
Staging & $1.77(0.64-4.84)$ & 0.27 \\
TP53 hotspot mutations & $3.57(1.44-8.86)$ & 0.006 \\
\hline
\end{tabular}

HR, hazard ratio; CI, confidence interval;

Table 6: Association between TP53 mutation in hot spots and recurrence in advanced GC

\begin{tabular}{lcc}
\hline Variables & TP53 hot spot mutations & Others \\
\cline { 2 - 3 } & $(\mathbf{n = 1 1 )}$ & $(\mathbf{n}=\mathbf{1 0 5})$ \\
\hline Over all recurrences n (\%) & $7(63.6)$ & $47(44.7)$ \\
Liver recurrences n (\%)\# & $3(27.3)$ & $8(7.6)$ \\
Peritoneal recurrences n (\%) & $3(27.3)$ & $32(30.5)$ \\
Lymph node recurrences n (\%) & $1(9.1)$ & $6(5.7)$ \\
Other recurrences n (\%)\$ & $3(27.3)$ & $6(5.7)$ \\
\hline
\end{tabular}

\#, $P<0.05 ; \$, P=0.02$;

biopsy samples, our data suggests the potential usefulness of endoscopic biopsy for the molecular analysis especially in patients with advanced stage who have no information from surgically resected specimens.

Among several clinicopathological features, TP53 mutation was more frequent in differentiated histologic type, which is in line with some previous reports [22, 23]. Of note, the correlation was striking among early stage, not observed in advanced stage. The data suggests that majority of TP53 mutated GC are differentiated histologic type in the early stage. It has been reported that progression of differentiated type GC often results in histological change to undifferentiated type [24]. It is therefore possible that considerable portion of TP53 mutated undifferentiated GCs may have originally developed as differentiated type. We also found that TP53 mutation was correlated with venous invasion in advanced GC. A similar trend was also found for more severe lymph node metastasis. This indicates mutation status reflects different biological behavior of GC. TP53 mutation has also been associated with more aggressive phenotypes of other tumor types, such as esophageal and colorectal cancers $[25,26]$. Since GC with venous invasion and severe lymph node metastasis often show more aggressive biological behaviors including relapsing even after curative surgery, TP53 mutated GC may need more intensive adjuvant chemotherapy or frequent follow up to prevent relapsing after gastrectomy.

We have also shown that subset of GCs with specific TP53 mutations (hot spots within the central core: R175, $\mathrm{G} 245, \mathrm{R} 248, \mathrm{R} 273, \mathrm{R} 282$ ) is associated with worse overall survival and recurrence free survival. The worse prognosis of these mutations was also confirmed as an independent factor using the multivariate survival analysis. The same mutation type was also associated with recurrences of GC. This result emphasizes the importance of discriminating 
TP53 mutations to divide GC patients for better clinical implementation reflecting biological behavior. Our data suggest that hotspot TP53 mutated GC may need more intensive therapy or careful follow up even after curative resection. Importance of discriminating TP53 change types is also reported in colorectal cancer patients with poor prognosis who have mutations in R175 and G245. Although the prevalence of these mutations are not so high in all GC in our dataset (13/214, 6.2\%). Significant worse prognosis of these patients suggests the potential usefulness of these mutations as a molecular marker in GC patients. Since the relationship between TP53 status and response to chemotherapy in $\mathrm{GC}$ patients has been conflicting [15-17], whether these mutations can predict response to chemotherapy needs to be evaluated in the larger cohort. We analyzed TP53 mutations in GC tissue, obtained by endoscopic biopsy. It should be noted that the results may not reflect the characteristics of whole part of the tumor considering the tumor heterogeneity. Still, our result showing the association between TP53 hotspot mutations and prognosis of GC would provide considerable information especially in patients who cannot undergo surgery due to the advance diseases.

\section{MATERIALS AND METHODS}

\section{Study population}

We enrolled 214 patients with gastric cancer (GC) treated in our hospital from September 2004 to February 2008. These patients consisted of 146 male and 68 females. Median age at diagnosis was 66 years (ranged from 29 to 94 years). All GC was diagnosed histologically. Detailed information about histologic subtypes, anatomic location, macroscopic types, lymph node and other metastasis and peritoneal dissemination was also obtained according to the Japanese classification of gastric carcinoma [18]. Information about treatment of GC was available for 210 patients. 172 patients performed gastrectomy with consideration of surgical indication, while 36 patients were diagnosed as non-resectable and were received chemotherapy. Two patients received best supportive care due to advanced diseases without indication of any curative treatment. Overall survival, defined as the time from gastrectomy, or start of initial administration of chemotherapy to the date of cancer related death was determined for 210 patients. Recurrence free survival, defined as the time from gastrectomy, or start of initial administration of chemotherapy to the date of tumor progression or cancer related death was also determined in the same cases. Patients with no confirmation of progression or cancer related death were censored at the date of the last objective tumor assessment. The Ethics Committee of Fujita Health University School of Medicine approved the protocol and written informed consent was obtained from all of the subjects.

\section{Sample collection, assessment of $H$. pylori infection}

During endoscopy, biopsy specimens were taken from primary GCs and adjacent normal-appearing mucosa. The specimens were immediately frozen and stored at $-80^{\circ} \mathrm{C}$ until use. Genomic DNA was extracted directly from these frozen specimens using the standard protein precipitation method. All DNA samples were obtained before gastrectomy or chemotherapy. Through histological examination of biopsy specimens taken almost same area of the tumor tissue, we confirmed that all biopsies from cancerous tissue contained more than $70 \%$ of cancer cells. H. pylori infection status was assessed by histological analysis of both the biopsy specimens from the greater curvature of the gastric antrum and upper corpus using antibodies.

\section{Sanger sequencing}

All coding regions of TP53 gene (exon2 to exon11) from GC tissues and matched normal gastric tissues were amplified using PCR reactions. These PCR fragments were sequenced using Sanger Sequencing. PCR and sequence primers are listed in Supplementary Table 1. The sequence chromatograms were visually inspected with DNA Dynamo Sequence Analysis Software (Blue Tractor Software, Llanfairfachan, Wales, UK). All mutations were confirmed by independent PCR and sequencing reactions. We only consider the nucleotide variation as the mutations, shown in the tumor samples, not in the normal tissues. For non-silent single nucleotide substitutions, SIFT (sorting intolerant from tolerant) analysis was performed to predict whether an amino acid substitution affects protein function.

\section{Statistics}

The statistical significance of the differential frequency of TP53 mutations in two groups was determined using two-tailed Fisher's exact test. Overall survival and recurrence free survival, in relation to the TP53 mutations was assessed using the KaplanMeier method and the Log rank test. $P$ value $<0.05$ was considered statistically significant.

\section{CONFLICTS OF INTEREST}

No conflicts of interest exist.

\section{GRANT SUPPORT}

This work was supported by JSPS KAKENHI Grantin-Aid for Young Scientists (B) Grant Number JP26870685. 


\section{REFERENCES}

1. Ferlay J, Bray F, Parkin DM, Pisani P, editors. Gobocan 2000: cancer incidence and mortality worldwide (IARC Cancer Bases No. 5). Lyon: IARC Press; 2001.

2. Lau M, Le A, El-Serag HB. Noncardia gastric adenocarcinoma remains an important and deadly cancer in the United States: secular trends in incidence and survival. Am J Gastroenterol. 2006;101:2485-2492.

3. Nashimoto A, Akazawa K, Isobe Y, Miyashiro I, Katai H, Kodera Y, Tsujitani S, Seto Y, Furukawa H, Oda I, Ono H, Tanabe S, Kaminishi M. Gastric cancer treated in 2002 in Japan: 2009 annual report of the JGCA nationwide registry. Gastric Cancer. 2013;16:1-27.

4. Kusano M, Toyota M, Suzuki H, Akino K, Aoki F, Fujita M, Hosokawa M, Shinomura Y, Imai K, Tokino T. Genetic, epigenetic, and clinicopathologic features of gastric carcinomas with the $\mathrm{CpG}$ island methylator phenotype and an association with Epstein-Barr virus. Cancer. 2006; 106:1467-79.

5. Clements WM, Wang J, Sarnaik A, Kim OJ, MacDonald J, Fenoglio-Preiser C, Groden J, Lowy AM. beta-Catenin mutation is a frequent cause of Wnt pathway activation in gastric cancer. Cancer Res. 2002;62:3503-6.

6. Li VS, Wong CW, Chan TL, Chan AS, Zhao W, Chu KM, So S, Chen X, Yuen ST, Leung SY. Mutations of PIK3CA in gastric adenocarcinoma. BMC Cancer. 2005;5:29.

7. Nakajima M, Sawada H, Yamada Y, Watanabe A, Tatsumi M, Yamashita J, Matsuda M, Sakaguchi T, Hirao T, Nakano H. The prognostic significance of amplification and overexpression of c-met and c-erb B-2 in human gastric carcinomas. Cancer. 1999;85:1894-902.

8. Wang K, Kan J, Yuen ST, Shi ST, Chu KM, Law S, Chan TL, Kan Z, Chan AS, Tsui WY, Lee SP, Ho SL, Chan $\mathrm{AK}$, et al. Exome sequencing identifies frequent mutation of ARID1A in molecular subtypes of gastric cancer. Nat Genet. 2011;43:1219-23.

9. Zang ZJ, Cutcutache I, Poon SL, Zhang SL, McPherson JR, Tao J, Rajasegaran V, Heng HL, Deng N, Gan A, Lim $\mathrm{KH}$, Ong CK, Huang D, et al. Exome sequencing of gastric adenocarcinoma identifies recurrent somatic mutations in cell adhesion and chromatin remodeling genes. Nat Genet. 2012;44:570-4.

10. Cancer Genome Atlas Research Network. Comprehensive molecular characterization of gastric adenocarcinoma. Nature. 2014;513:202-9.

11. Kakiuchi M, Nishizawa T, Ueda H, Gotoh K, Tanaka A, Hayashi A, Yamamoto S, Tatsuno K, Katoh H, Watanabe Y, Ichimura T, Ushiku T, Funahashi S, et al. Recurrent gain-of-function mutations of RHOA in diffuse-type gastric carcinoma. Nat Genet. 2014;46:583-7.

12. Lamb P, Crawford L. Characterization of the human $\mathrm{p} 53$ gene. Mol Cell Biol. 1986;6:1379-85.

13. Vousden KH, Prives C. Blinded by the Light: The Growing Complexity of p53. Cell. 2009;137:413-31.
14. Fenoglio-Preiser CM, Wang J, Stemmermann GN, Noffsinger A. TP53 and gastric carcinoma: a review. Hum Mutat. 2003;21:258-70.

15. Fareed KR, Al-Attar A, Soomro IN, Kaye PV, Patel J, Lobo DN, Parsons SL, Madhusudan S. Tumour regression and ERCC1 nuclear protein expression predict clinical outcome in patients with gastro-oesophageal cancer treated with neoadjuvant chemotherapy. Br J Cancer. 2010;102:1600-7.

16. Ott K, Vogelsang H, Mueller J, Becker K, Müller M, Fink U, Siewert JR, Höfler H, Keller G. Chromosomal instability rather than 53 mutation is associated with response to neoadjuvant cisplatin-based chemotherapy in gastric carcinoma. Clin Cancer Res. 2003;9:2307-15.

17. $\mathrm{Xu} \mathrm{HY}, \mathrm{Xu}$ WL, Wang LQ, Chen MB, Shen HL. Relationship between p53 status and response to chemotherapy in patients with gastric cancer: a metaanalysis. PLoS One. 2014;9:e95371.

18. Japanese Gastric Cancer Association. Japanese classification of gastric carcinoma: 3rd English edition. Gastric Cancer. 2011;14:101-12.

19. Nguyen T, Brunson D, Crespi CL, Penman BW, Wishnok JS, Tannenbaum SR. DNA damage and mutation in human cells exposed to nitric oxide in vitro. Proc Natl Acad Sci U S A. 1992;89:3030-4.

20. Wink DA, Kasprzak KS, Maragos CM, Elespuru RK, Misra M, Dunams TM, Cebula TA, Koch WH, Andrews AW, Allen JS. DNA deaminating ability and genotoxicity of nitric oxide and its progenitors. Science. 1991;254:1001-3.

21. Sugimura T, Kawaki T. Experimental stomach cancer. Methods Cancer Res 1973;7:245-308.

22. Wu MS, Shun CT, Wang HP, Sheu JC, Lee WJ, Wang TH, Lin JT. Genetic alterations in gastric cancer: relation to histological subtypes, tumor stage, and Helicobacter pylori infection. Gastroenterology. 1997;112:1457-65.

23. Wu MS, Shun CT, Sheu JC, Wang HP, Wang JT, Lee WJ, Chen CJ, Wang TH, Lin JT. Overexpression of mutant p53 and c-erbB-2 proteins and mutations of the p15 and p16 genes in human gastric carcinoma: with respect to histological subtypes and stages. J Gastroenterol Hepatol. 1998;13:305-10.

24. Ikeda Y, Mori M, Kamakura T, Haraguchi Y, Saku M, Sugimachi K. Increased incidence of undifferentiated type of gastric cancer with tumor progression in 912 patients with early gastric cancer and 1245 with advanced gastric cancer. Cancer. 1994;73:2459-63.

25. Madani K, Zhao R, Lim HJ, Casson AG. Prognostic value of p53 mutations in oesophageal adenocarcinoma: final results of a 15-year prospective study. Eur J Cardiothorac Surg. 2010;37:1427-32.

26. Lleonart ME, García-Foncillas J, Sánchez-Prieto R, Martín P, Moreno A, Salas C, Ramón y Cajal S. Microsatellite instability and p53 mutations in sporadic right and left colon carcinoma: different clinical and molecular implications. Cancer. 1998;83:889-95. 